\title{
Experimental Study on Response Sensitivity of Smoke Detectors in High Flow Velocity
}

\author{
QIYUAN XIE ${ }^{1}$, GUOFENG SU ${ }^{2}$, HONGYONG YUAN ${ }^{2}$, and YONGMING ZHANG ${ }^{1}$ \\ ${ }^{1}$ State Key Laboratory of Fire Science \\ University of Science and Technology of China \\ Hefei, 230027, P.R. China \\ ${ }^{2}$ China Center for Public Safety Research, Tsinghua University \\ Beijing, 100084, P.R. China
}

\begin{abstract}
The objective of this work was to investigate preliminary design criteria of automatic fire alarm systems for some places with high flow velocity, e.g., exits of large underground car parks, the air outlets of large air conditioner etc. In addition, another main motivation of this research is to evaluate the appropriateness of Chinese standard which states that photoelectrics should be used in high flow locations. Two corresponding series of experiments were designed to analyze the response sensitivity of photoelectric and ionization smoke detectors under the conditions of different flow velocities in the Fire Emulator/Detector Evaluator (FE/DE), using smoldering cottons as the smoke source. The combining analysis of results of the two series of experiments indicated that generally speaking, ionization smoke detectors showed better response sensitivity than photoelectric smoke detectors in environments with high flow velocity. Preliminary analysis suggested that the influence of flow velocity on size distribution of the smoke particles generated is the main reason for the results.
\end{abstract}

KEYWORDS: flow velocity, smoke detectors, smoke aerosol, size distribution

\section{INTRODUCTION}

The smoke aerosol is motivated by the buoyancy force when fires happen in common places with small air velocity [1,2]. Therefore, the fire detectors mounted there are required to have good smoke sensitivity under the condition of low flow velocity. However, in some special places, such as exits of large underground car parks, and the air outlets of large air conditioner, the flow velocity may be much higher. When a fire happens in such places, its combustion behavior and the induced characteristics (e.g., size distribution, color, etc.) of smoke particles generated would be quite different from those generated under the conditions of low air velocity. Accordingly, it is required that the fire detectors mounted there should have good smoke sensitivity in the environments with high flow velocity. In this case, the response sensitivity of smoke detectors is influenced by not only the smoke density around them, but also the size distribution of particles, as well as smoke particles' entry into the smoke-sensing-chamber of detector in high flow velocity. After investigating and analyzing the scenarios with high flow velocities, it is found that the influence of high flow velocity on the response sensitivity of smoke detectors falls into two types. First, due to the high flow velocity around detectors, the smoke particles pass by fire detectors with a high velocity. Therefore, in fact, the affects of flow velocity here include the dilution of smoke density around detectors, and smoke particles' entry into the smoke-sensing-chamber of detectors. Accordingly, experiments should be conducted to investigate the response sensitivity of 
smoke detectors using same smoke particles with different flow velocity around detectors. Secondly, the flow velocity not only around detectors but also around smoke source may be high. In this case, since the smoke generation is effected by the flow velocity around burning material, the characteristics, e.g., size distribution, of smoke particles generated are strongly related to the flow velocity. Additionally, the response sensitivities of various kinds of smoke detectors, e.g., photoelectric and ionization smoke detectors, to the smoke aerosol with different characteristics are different. Thomas Cleary [3] and Keski Rahkonen [4] had studied time-lag models of smoke detectors under the conditions of low velocity. But the response sensitivity of smoke detectors in high flow velocity was few involved in literatures.

In accordance with the two aspects of influence of flow velocity on the response sensitivity of smoke detectors, two series of corresponding experiments were designed to analyze the two kinds of most widely used smoke detectors, i.e., photoelectric and ionization smoke detectors, in various flow velocities through the Fire Emulator/Detector Evaluator (FE/DE). The basic aim of the present work is to give preliminary design criteria of automatic fire alarm systems in those places with high flow velocity. In addition, it is useful to evaluate whether it is appropriate to choose the photoelectric smoke detectors in high flow locations, as stated in Chinese standard [5].

\section{EXPERIMENTAL APPARATUS}

All the experiments were conducted in the FE/DE, which was developed by State Key Laboratory of Fire Science (SKLFS) in University of Science and Technology of China (USTC). The FE/DE is for emulating a wide range of fire and non-fire scenario to which a spot-type fire detector could be exposed. The concept of FE/DE is first introduced by Grosshandler [6]. The FE/DE in the SKLFS is a $0.4 \mathrm{~m}$ high by $0.4 \mathrm{~m}$ wide cross-section flow tunnel designed to reproduce the time-varying temperature, speed and concentration (gas and particulate) expected at detector locations in the early stages of fire (Fig. 1a). The inside and outside walls of the tunnel are made of stainless steel, and the interlayer is the asbestos, which is heat insulating. The FE/DE employs a variable speed blower to control velocities ranging from $0.02 \mathrm{~m} / \mathrm{s}$ to greater than $5 \mathrm{~m} / \mathrm{s}$. The blower is controlled by the transducer with a feed-back velocity. Water mist produced by the centripetal humidifier may be sprayed into the flow after the heater section to fix the humidity between the ambient room and saturation conditions depending on the spray flow. CO, $\mathrm{CO}_{2}$ or other gas blends may be introduced into the flow via electronic mass controllers. Not only the fire scenario but also the nuisance alarm scenario can be emulated in the $\mathrm{FE} / \mathrm{DE}$. The dust with different diameters may be metered into the flow. As is shown in Fig. 1a, near the entrance of the flow tunnel, there is a relatively hermetic box for smoke-generating. Figure 1a also shows that the apparatus can work at either open-style or circle-style by merely changing the states of the three valves. In this paper, the open-style work-mode is selected during all the experiments. In this case, smoke aerosol can be generated through the box with smoke source inside the box, as the location A indicated in the Fig. 1a. Alternatively, the smoke can be generated openly at the entrance of the flow tunnel with smoke source at the location B in Fig. 1a. Two series of experiments were designed in terms of the two locations of smoke source, which will be further described later. As is mentioned above, the FE/DE at SKLFS can be operated in the open-style and circle-style work-modes, which can be easily switched to each other. Here lies the most distinct difference between the FE/DE at the SKLFS and the one at 
NIST $[7,8]$. The adding of a circle-style work-mode in the SKLFS has made it easier to simulate the processes of increasing of some fire signals (smoke or gas concentration, temperature etc.) in a wider range.

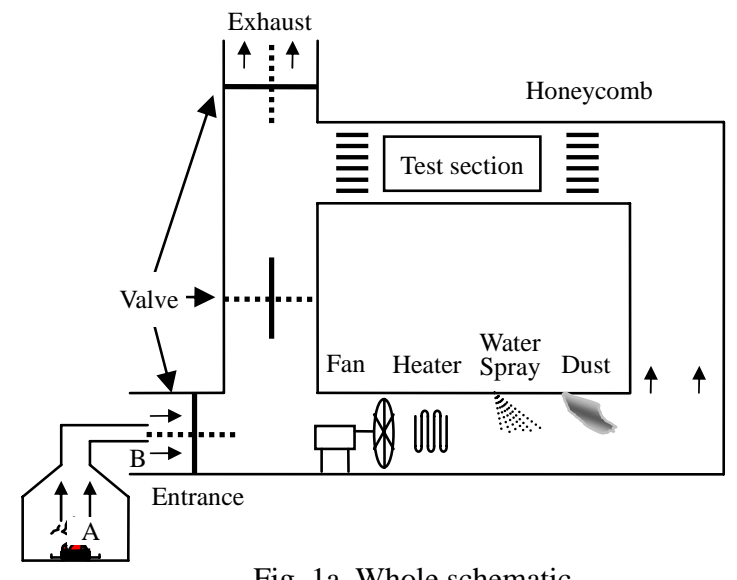

Smoke box
Fig. 1a. Whole schematic.

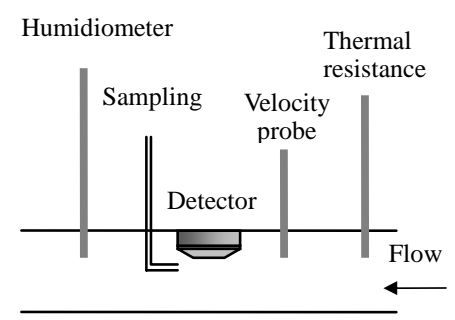

Fig. 1b. Side view of test section.

Fig. 1. The schematic of Fire Emulator/Detector Evaluator. Valves located at the dashed line state: open-style work-mode. Valves located at the real line state: circle-style work-mode.

The spot-type fire detectors to be evaluated, as well as the measurement instruments, were mounted at the test section (see Fig. 1b). Here commercially available digital addressable point smoke detectors were used, including forward scattering photoelectric detectors and ionization detectors. The digital output value of a detector ranges from 0 to a maximum of 255 , relatively corresponding to the intensity of scattered light in the forward angle, i.e., the smoke density. A mapping transformation between the digital outputs and the real smoke density is defined by the manufacture. The detector manufacturer's instruction book recommended that a threshold level of 115 units for photoelectric and 170 units for ionization would be appropriate fire alarm triggers with common sensitivity, respectively. The real-time outputs of the smoke detectors were displayed and recorded through the communication between the control panel and the personal computer using a terminal program. Air temperature, humidity and velocity were recorded at the test section by thermal resistances, humidiometer and thermo-anemometer, respectively. For more information about this experimental apparatus, please refer to references $[9,10,11]$.

\section{EXPERIMENTS DESCRIPTION}

As is introduced above, the process of smoke generating, and the induced characteristics of smoke aerosol, may be differently influenced by the flow velocity under different environmental conditions. Experiments should be respectively designed to investigate the two types of effects of flow velocity on the response sensitivity of smoke detectors. Therefore, here two series of experiments were conducted to analyze the response sensitivity of photoelectric and ionization smoke detectors under different flow 
velocities. Additionally, further comparison of the response sensitivity of the two kinds of smoke detectors was also made when the flow velocity was as high as $5.0 \mathrm{~m} / \mathrm{s}$. The results would be preliminary criteria for the design of automatic fire alarm systems for some places with high flow velocity. In terms of this idea, two series of experiments were designed respectively as the follows (a) and (b).

(a) 60 smoldering standard cottons [12] were set at location A, i.e., in the smoke-generating-box, as indicated in Fig. 1a. The same smoke aerosol would be generated in the box and then released into flow tunnel, acting as the smoke source under the conditions of different flow velocities. The smoldering cottons were taken away after burning in the box for about 5 minutes. In this case, 11 sets of experiments were carried out with the flow velocity in the tunnel $0.5 \mathrm{~m} / \mathrm{s}, 1.0 \mathrm{~m} / \mathrm{s}$, $\ldots, 5.5 \mathrm{~m} / \mathrm{s}$, respectively.

(b) 60 smoldering standard cottons were put at location B, as indicated in Fig. 1a. The smoke aerosol was openly generated at the entrance of the FE/DE. The same with (a), the smoldering cottons were taken away after they had burnt at the entrance of the FE/DE for about 5 minutes. Also, 11 sets of experiments were carried out with the flow velocity in the tunnel $0.5 \mathrm{~m} / \mathrm{s}, 1.0 \mathrm{~m} / \mathrm{s}, \ldots, 5.5 \mathrm{~m} / \mathrm{s}$, respectively.

All the experiments above were carried out at the open-style work-mode of the FE/DE. The only difference of experimental conditions among the 11 sets of experiments for (a) or (b) lies in the flow velocity in the tunnel. Therefore, the first step of each set of experiment is to alter the rotate speed of the fan, for adjusting the flow velocity in the test section of the FE/DE. Here, altogether 22 sets of experiments should be conducted for the two series of experiments. For each set of experiment two runs were performed under the same experimental conditions, and the experiment reproducibility was verified by reasonable agreement between the two obtained curves of outputs of corresponding detectors.

With regards to experiment series (a), there was no difference in the characteristics of the smoke aerosol generated among the 11 sets of experiments with different flow velocity in the tunnel. The reason is that smoke aerosol was generated inside the relatively hermetic box and the flow velocity in the tunnel would not affect the process of smoke generating. However, for the experiment series (b), since the smoldering cottons were openly put at the entrance of the FE/DE, the process of smoke generation was influenced by the flow velocity in the tunnel. Therefore, the characteristics, for example the size distribution, of smoke aerosol here were somewhat different among the 11 sets of experiments with different flow velocity.

\section{RESULTS AND DISCUSSION}

For the experiment series (a), Figs. 2-4 give the outputs of photoelectric and ionization smoke detectors with the flow velocity in the test section of $1.0 \mathrm{~m} / \mathrm{s}, 2.0 \mathrm{~m} / \mathrm{s}$, and 3.0 $\mathrm{m} / \mathrm{s}$, respectively. 
Figures 2-4 show that both peak outputs of two kinds of smoke detectors decreased while the flow velocity in the tunnel increasing from $1.0 \mathrm{~m} / \mathrm{s}$ to $3.0 \mathrm{~m} / \mathrm{s}$. That is to say, the corresponding increments of outputs decreased. Here, the maximum output minus the minimum output yields the 'increment of output' of a smoke detector under a certain experimental condition. The peaking of the curves in these figures is a result of the flow of a finite amount of smoke past the detectors. The response sensitivity of fire detectors depends on their changes of outputs and the corresponding fire alarm algorithms. Therefore, the increments of outputs could represent the response sensitivity of detectors to some degree. For experiments series (a), Fig. 5 showed the increments of outputs of photoelectric and ionization smoke detectors with the flow velocity $0.5 \mathrm{~m} / \mathrm{s}, 1.0 \mathrm{~m} / \mathrm{s}, \ldots$, $5.5 \mathrm{~m} / \mathrm{s}$, respectively. As can be seen from Fig. 5, it was more clearly that increments of outputs of both the two kinds of smoke detectors fell down with the flow velocities increasing. The reason for the above results can be qualitatively analyzed as follows. Not only the amount, but also the characteristics, e.g., size distribution etc, of smoke particles was the same among the 11 sets of experiments with different flow velocities. The main influence of flow velocity here is the dilution of smoke density in the tunnel. In fact, the higher flow velocity in the tunnel, the smaller corresponding smoke density. Additionally, there also exists the affect of flow velocity on the particles entry of smoke detectors. But this affect is too weak to be compared with the dilution on smoke density. Consequently, the increments of outputs of two kinds of smoke detectors decreased with the flow velocities increasing, namely, the corresponding response sensitivity decreased.

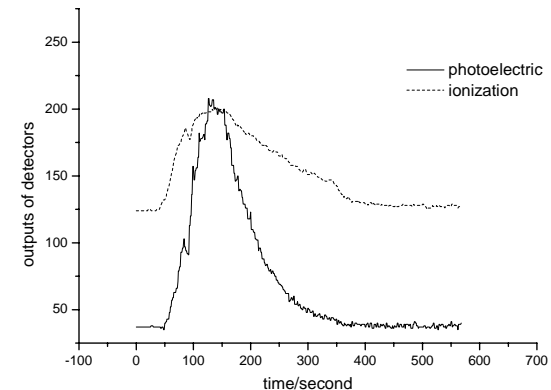

Fig. 2. Outputs of detectors $(1.0 \mathrm{~m} / \mathrm{s}$, box $)$.

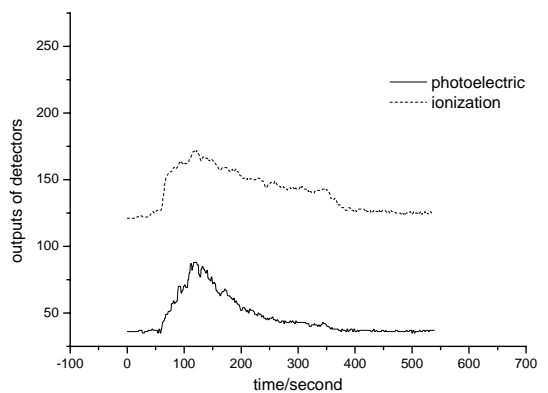

Fig. 3. Outputs of detectors $(2.0 \mathrm{~m} / \mathrm{s}$, box $)$.

For the experiment series (b), Figs. 6-8 show the outputs of photoelectric and ionization smoke detectors under the condition of the flow velocity in test section $1.0 \mathrm{~m} / \mathrm{s}, 2.0 \mathrm{~m} / \mathrm{s}$, and $3.0 \mathrm{~m} / \mathrm{s}$, respectively. 


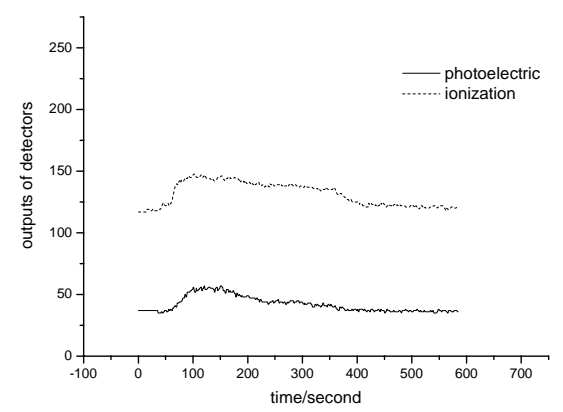

Fig. 4. Outputs of detectors $(3.0 \mathrm{~m} / \mathrm{s}$, box $)$.

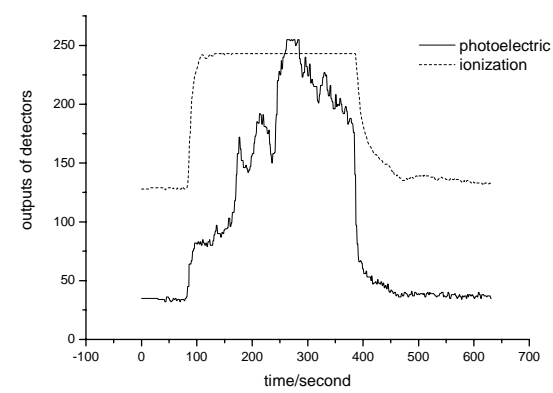

Fig. 6. Outputs of detectors (1.0 m/s, entrance).

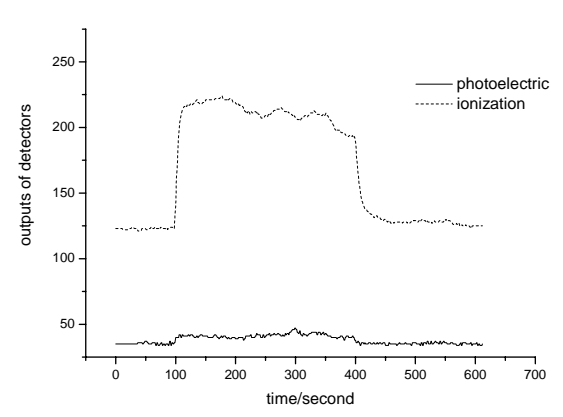

Fig. 8. Outputs of detectors (3.0 $\mathrm{m} / \mathrm{s}$, entrance).

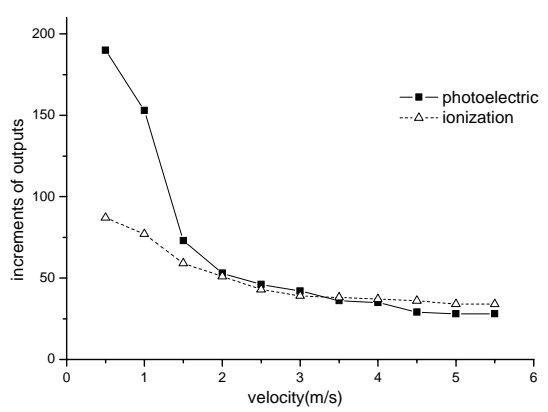

Fig. 5. Increments of outputs (box).

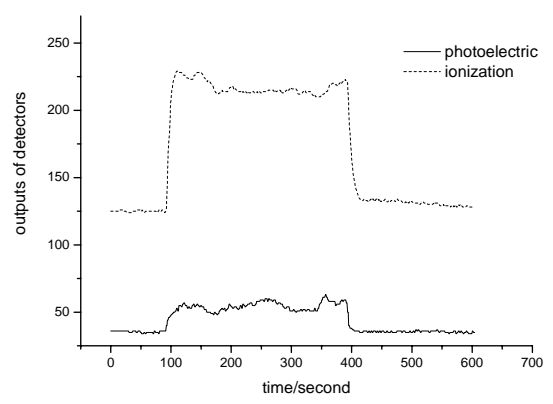

Fig. 7. Outputs of detectors ( $2.0 \mathrm{~m} / \mathrm{s}$, entrance).

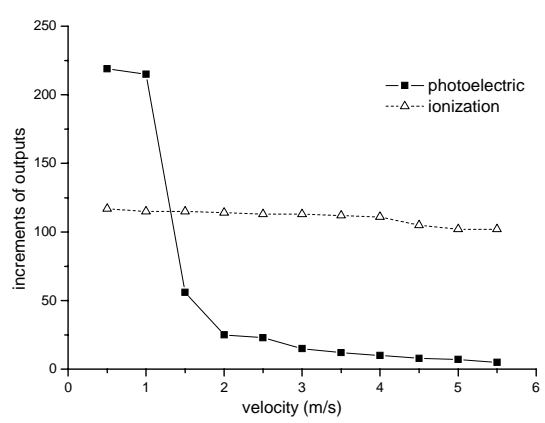

Fig. 9. Increments of outputs (entrance). 
As can be seen from Figs. 6-8, the experiment results here were considerably different from those in experiment series (a). The corresponding 'increments of outputs' of both photoelectric and ionization smoke detectors decreased with the flow velocity increasing in series (a). However, in experiments series (b), as shown in Figs. 6-8, only the 'increments of the outputs' of photoelectric smoke detectors decreased sharply when the flow velocities increased. The corresponding increments of outputs of ionization detectors hardly decreased. First, let's compare and analyze the three output-curves of photoelectric smoke detectors in Figs. 6-8, with the flow velocity of $1.0 \mathrm{~m} / \mathrm{s}, 2.0 \mathrm{~m} / \mathrm{s}$ and $3.0 \mathrm{~m} / \mathrm{s}$, respectively. As shown in Fig. 6, the outputs of photoelectric smoke detectors increased steeply after the smoldering cottons being put in, with the flow velocity 1.0 $\mathrm{m} / \mathrm{s}$. But the corresponding curve of photoelectric detectors in Fig. 7 seemed to be much flater with the flow velocity $2.0 \mathrm{~m} / \mathrm{s}$. Furthermore, the increase of the outputs of photoelectric smoke detectors can be hardly noticed with the flow velocity $3.0 \mathrm{~m} / \mathrm{s}$ in Fig. 8. After the comparison of the three curves for photoelectric smoke detectors, let's turn to analyze the other three curves of ionization smoke detectors in Figs. 6-8. It is obvious that the corresponding outputs increased sharply as soon as the smoldering cottons were set at the entrance of the FE/DE in all the three curves. The increments of outputs of ionization smoke detectors seemed to be slightly influenced by the flow velocity in the tunnel under this condition. The different variation of the increments of outputs between the photoelectric and ionization smoke detectors can be observed more clearly in Fig. 9. Here, it should be particularly pointed out that the ionization smoke detectors still responded sensitively while the photoelectric smoke detectors responded little with the flow velocity as high as $5.5 \mathrm{~m} / \mathrm{s}$.

The reasons for the results of experiment series (b) could be analyzed as follows. Since the smoldering cottons were put openly at the entrance of the FE/DE, the process of smoke generating was influenced by the flow velocity around. First, the burning rate of the smoldering cottons was affected by the flow velocity in the tunnel. The burning rate of the smoldering cottons would become larger when the flow velocity around increased. Namely, the more smoke particles would be generated per unit time. Secondly, the combustion behavior of smoldering cottons was also influenced by the flow velocity around. Increasing the flow velocity in the vicinity of smoldering cottons decreased particles size. The reason is that it decreases the residence time for particles to grow by coagulation [13]. So the size distribution of smoke particles generated would shift towards the smaller side with the flow velocity increasing. Therefore, the mean diameter of smoke particles decreased. Additionally, with different flow velocities, the dilution effect on the smoke density in the tunnel was also different. The influence of flow velocity on the size distribution of smoke particles generated was the key reason for the results in experiment series (b). It is also important to point out that ionization smoke detectors generally responded much more sensitively to smaller smoke particles than photoelectric smoke detectors $[14,15,16]$. So the experiment series (b) suggested that the ionization smoke detectors showed better response sensitivity than photoelectric smoke detectors under the condition of high flow velocity. However, the size distributions of smoke particles generated from different fuels vary distinctly. For example, the Mass Median Aerodynamic Diameter (MMAD) of cotton Lamp Wick is 0.31 um, other fuels such as Beech Wood and Heptane present pretty larger particle size with the MMAD of $1.5 \mathrm{um}$ and $6.4 \mathrm{um}$, respectively [17]. In this case, the measurement of the size 
distribution of particles at detector location with different flow velocity will be an important topic of future work.

The above preliminary experiments suggest that the ionization smoke detectors showed better response sensitivity than photoelectric smoke detectors in high flow velocity with the smoke particles generated by smoldering cottons. However, the use of radioactive material for ionization smoke detectors has been more regulatory controlled and restricted for its use and handling as years go by. Photoelectric smoke detectors are currently taking over the place once ionization smoke detectors held in the market. Therefore, it is necessary to do further research on the interaction between smoke particles and light, for the aim of improving the response sensitivity of photoelectric smoke detectors in some special environments.

\section{CONCLUSION}

In this paper, effect of flow velocity on response sensitivity of photoelectric and ionization smoke detectors has been analyzed with two series of experiments. Based on analysis for results of the two series of experiments with smoke particles generated by smoldering cottons in this paper, it preliminarily suggests that ionization smoke detectors responded generally more sensitively than the photoelectric smoke detectors under the condition of high flow velocity. But the code for design of automatic fire alarm system (GB50116-98, Chinese standard code) recommends that photoelectric smoke detectors should be chosen in the places with air velocity exceeding $5.0 \mathrm{~m} / \mathrm{s}$ [5]. Namely, the experimental results in this article were surprisingly different to the code. Since the particles characteristics (number, size and distribution) are important factors influencing the response sensitivity of smoke detectors, additional measurement of particle characteristics at detector location with different flow velocity will be done in the future work. The other different fuels such as Heptane or wood and more smoke detectors produced by other companies should also be considered.

\section{ACKNOWLEDGMENTS}

This paper is supported by the China NKBRSF (973) project (No: 2001CB409608) and the National Natural Science Foundation of China (Grant No.50476023). The authors deeply appreciate their support.

\section{REFERENCES}

[1] Chow, W.K, and Yin, R., "Free Boundary Conditions for Simulating Air Movement in a Big Hall Induced by a "Bare Cabin" Fire," Journal of Fire Sciences, 17: 111-147, 1999.

[2] Cooper, L.Y., "Calculating Combined Buoyancy - and Pressure-driven Flow Through a Shallow, Horizontal, Circular Vent: Application to a Problem of Steady Burning in a Ceiling-vented Enclosure," Fire Safety Journal, 27: 23-35, 1996.

[3] Cleary, T. G., Chernovsky, A., Grosshandler, W. L. and Anderson, M., "Particulate Entry Lag in Spot-Type Smoke Detectors," Proceedings of 6th International Symposium, IAFSS, 2000, pp. 779-790. 
[4] Keski Rahkonen, O., "Revisting Modelling of Fluid Penetration in to Smoke Detectors Revisited for Low Speed Ceiling Jets," Proceedings of the 12th International Conference on Automatic Fire Detection "AUBE '01," National Institute of Standards and Technology, 2001, pp. 506-516.

[5] GB50116-98 Standard of the Design of Automatic Fire Alarming Systems[S], 1998.

[6] Grosshandler, W.L., "Towards the Development of a Universal Fire Emulator/ Detector Evaluator,” Fire Safety Journal, 29, 113-128, 1997.

[7] Cleary, T., and Donnelly, M., and Grosshandler, W., "The Fire Emulator/Detector Evaluator: Design, Operation, and Performance,” Proceedings of the 12th International Conference on Automatic Fire Detection "AUBE '01," National Institute of Standards and Technology, pp. 312-323, 2001.

[8] Cleary, T., Grosshandler, W., and Chernovsky, A., "Smoke Detector Response to Nuisance Aerosols," Proceeding of the 11th International Conference on Automatic Fire Detection "AUBE '99," Gerhard Mercator University, pp. 32-41, 1999.

[9] Yuan Hongyong, Xie Qiyuan, Su Guofeng, Zhan Furu, Zhao Jianhua, and Fan Weicheng, "A Kind of Fire Emulator/Detector Evaluator," Chinese patent: ZL 02219803.2, 2003.

[10] Xie Qiyuan, Zhan Furu, Su Guofeng, Yuan Hongyong, and Zhao Jianhua, "Universal Simulated Platform for Fire Detection," Fire Safety Science, 11(2): 98-102 (2002).

[11] Xie Qiyuan, Yuan Hongyong, and Guo Huiliang, "Experimental Analysis on False Alarms of Fire Detectors by Cooking Fumes," Journal of Fire Sciences, 22, 4, 325-338, 2004.

[12] EN54: Compartments of Automatic Fire Detection Systems, European committee for standardization, 1987.

[13] Lee, T.G.K. and Mulholland, G., "Physical properties of Smoke Pertinent to Smoke Detector Technology,” NBSIR, pp. 77, 1977.

[14] Fleming, J., "Photoelectric vs. Ionization Detectors - A Review of the Literature,” Proceedings Fire Suppression and Detection Research Application Symposium, Natl. Fire Protection Association, pp. 18-59, 1998.

[15] Qualey, J., Desmarais, L., and Pratt, J., "Fire Test Comparisons of Ion and Photoelectric Smoke Detector Response Times," Fire Suppression and Detection Research Application Symposium, 2001, Orlando, FL.

[16] Muholland, G., "Smoke Production and Properties," The SFPE Handbook of Fire Protection Engineering (2nd Ed), DiNenno P.J. (ed.), National Fire Protection Association, Quincy, MA 02269, P.2/221-222, 1995. 
[17] Weinert D.W., Cleary T.G., Mullolland, G.W., and Beever, P.F., "Light Scattering Characteristics and Size Distribution of Smoke and Nuisance Aerosols," Fire Safety Science Proceedings, Seventh International Symposium, International Association for Fire Safety Science (IAFSS), Worcester, MA, pp.209-220, 2003. 